\title{
Portable Leaf Area Meter: A Review
}

\author{
Lakshmy Chandran $\mathrm{S}^{1}$, Parveez Shariff $\mathrm{B}^{2}$, Praveen $\mathbf{J}^{3}$, Raghavendra Rao $\mathrm{A}^{\mathbf{4}}$ \\ M.Tech Student, Dept of ECE, VLSI Design and Embedded Systems, Mijar, Karnataka, India ${ }^{1}$ \\ Assistant Professor, Dept of ECE, AIET, Mijar, Karnataka, India ${ }^{2}$ \\ Sr. Associate Professor, Dept of ECE, AIET, Mijar, Karnataka, India ${ }^{3,4}$
}

\begin{abstract}
This paper is about designing a portable leaf area meter using Beagle board single board computer and a line scan image sensor. A leaf area meter is a device used by agriculture scientists as well as in agriculture industry to measure the area of leaves.

The goal of this project is to design a portable system more like a hand held scanner that can be used to scan a leaf and get a measurement of its area, size and shape and optionally diseased areas also. The system will be designed around a beagle board single board computer interfaced to a line scan image sensor and also a LCD/LED display panel and a touch keypad. The system will be handheld or dockable to a portable conveyor. Results will be stored on-board or can be transferred via optionally wireless mode to a desktop machine.
\end{abstract}

Keywords: BeagleBoard, BeagleBoneBlack, Line scan sensor, Optical encoder, Edge detection

\section{INTRODUCTION}

The proposed system is application specific, which is widely used by agricultural scientists and genetic engineers who works on producing new plants in entomology, crop physiology, etc to detect the insect damage, growth rate of the plant, to analyse and check if the spacing between each plant in a field is optimum, to determine the consumption of energy from sun, to analyse the growth rate of each plant with respect to their leaves, etc. Thus this system is having numerous applications in this area. The proposed model could detect the leaf area length, width, any hole or defected area in the shape of the leaf. This is having more application in plant genetic engineering.

The proposed system is employed using the most advanced version of Beagle Board, that is BeagleBoneBlack and a line scan image sensor.

The Beagle Board is a low-power open-source hardware single-board computer produced by Texas Instruments in association with Digi-Key and Newark element. The BeagleBoard was also designed with open source software development in mind.

Here we are using the BeagleBoneBlack Rev C. The sensor used is Hamamatsu S11108 line scan CMOS linear sensor for capturing the image as line arrays of pixels. We use a BeagleBoneBlack expansion LCD display cape for the display of output. We have to use an optical encoder to track and synchronise the sensor integration speed with the scanning speed. To make the system portable, we are using a polymer lithium ion battery to power the system.

\section{LITERATURE SURVEY}

[1] presents the development of a solar cell based simple Some sensor architecture comparison and the logic behid and practical leaf area meter. The system has a silicon its working is explained in [3] by Dalsa. It also describes solar module which is composed of 6 series connected the basic idea behind line scan imaging and also its cells, a light source, and a parabolic reflector with opal difference with area scan imaging. It also gives an glass for diffusing the light. Partial shading effect on PV example application with an encoder. To find more about cell's voltage and current are evaluated with different how to control the exposure time of the sensor, this paper geometrical shapes for testing the performance of the could be very helpful. model, and the resulting equations for voltage and current 
[4] describes about the working of incremental and S11108 is a CMOS linear image sensor that achieves high absolute encoders. The difference between absolute and sensitivity by adding an amplifier to each pixel as incremental encoders is clearly and briefly defined in this explained in [8]. It has a long photosensitive area paper. This helped me to decide which type of encoder has to be used in my project. With the help of timing diagrams the working of the encoders is described well in this paper. It also gives idea about the encoder quality, loads given to encoders, loads that can be given to encoders, heavy loads, operating temperature conditions preferable for encoders, different working environments possible for encoders,etc.

[5] describes specifically about absolute encoders. The Optical Encoders typically consist of a rotating and a stationary member. The rotor is usually a metal, glass, or a plastic disc mounted on the encoder shaft. The disc has some kind of optical pattern, which is electronically decoded to generate position information. The rotor disc in absolute optical encoder uses opaque and transparent segments arranged in a gray-code pattern. The stator has corresponding pairs of LEDs and phototransistors arranged so that the LED light shines through the transparent sections of the rotor disc and received by phototransistors on the other side. After the electronic signals are amplified and converted, they are then available for the evaluation of the position.

In order to meet the demand of real-time spatial data processing and improve the online processing capability of photogrammetric system, a kind of real-time photogrammetry method is proposed in this paper. According to the proposed method in [6], system based on embedded architecture is then designed: using FPGA, ARM+DSP and other embedded computing technology to build specialized hardware operating environment, transplanting and optimizing the existing photogrammetric algorithm to the embedded system, and finally real-time photogrammetric data processing is realized. At last, aerial photogrammetric experiment shows that the method canachieve high-speed and stable on-line processing of photogrammetric data. And the experiment also verifies the feasibility of the proposed real-time photogrammetric system based on embedded architecture. It is the first time to realize real-time aerial photogrammetric system, which can improve the online processing efficiency of photogrammetry to a higher level and broaden the application field of photogrammetry.

[7] gives the detailed documents regarding 4.3" LCD CAPE of Beagle Bone Black. The 4D 4.3" LCD CAPE is a cape specifically designed for the Beagle Bone Black (BBB), and provides a 4.3 " primary display for the BBB for direct user interaction and information display. It is available in both touch (4DCAPE-43T) and non-touch (4DCAPE-43). The 4DCAPE-43 is not compatible with the previous Beagle Bone (Beagle Bone White), and can only be used with the Beagle Bone Black. The 4D 4.3" LCD CAPE features a 4.3" TFT LCD 480x272 resolution display. It has 7 push buttons for the user's convenience.

(effective photosensitive area length: 28.672mm) consisting of 2048 pixels, each with a pixel size of $14 \times 14$ $\mu \mathrm{m}$. The full electrical and optical characteristics of the sensor is explained in it. While the operation can be done in two ways. One as outputting signals from 2048 channels and the other by outputting signals from 1024 channels.

AM335x is the processor used in Beagle Bone Black. It has high speed for processing as in [9]. It is an ARM Cortex A8 microprocessor. It is a 32 bit RISC processor. It has Crypto hardware accelerators. It also has 12-bit Successive Approximation Register (SAR) ADC. Two Controller Area Network (CAN) ports are also available for it. It has MPU subsystems. ON-Chip memory, L3 RAM is provided. External Memory Interfaces (EMIF), General Purpose Memory Controller (GPMC), Error Locator Module (ELM), Power Reset and Clock Management (PRCM) Module, Real Time Clock for real time applications, Peripherals like two USB2.0 ports, two CAN ports, etc, LCD controller, 12-Bit SAR ADC, Debug Interface Support, DMA, Inter Processor Communication (IPC), etc. Its applications are gamming peripherals, home and industrial automation, consumer medical appliances, printers, smart toll systems, connected vending machines, weighing scales, educational consoles and advanced toys. This device supports only the following high level operating systems like Linux ${ }^{\circledR}$, Windows ${ }^{\circledR} \mathrm{CE}$, Android ${ }^{\mathrm{TM}}$.

[10] gives information regarding how to use Beagle Bone Black(BBB), how to connect it to PC, how to power it, and other connections possible for it. It even helps in booting scenario. It tells us about all the internal as well as external connections in it. Processors and other ICs used in $\mathrm{BBB}$, while booting and initialising the instructions and coding required, many ports available and its uses are all given in it. The Beagle Bone Black is the latest addition to the BeagleBoard.org family and like its predecessors, is designed to address the Open Source Community, early adopters, and anyone interested in a low cost ARM Cortex-A 8 based processor. It has been equipped with a minimum set of features to allow the user to experience the power of the processor and is not intended as a full development platform as many of the features and interfaces supplied by the processor are not accessible from the Beagle Bone Black via on-board support of some interfaces. It is not a complete product designed to do any particular function. It is a foundation for experimentation and learning how to program the processor and to access the peripherals by the creation of your own software and hardware. It also offers access to many of the interfaces and allows for the use of add-on boards called capes, to add many different combinations of features. A user may also develop their own board or add their own circuitry.

Hand-held leaf area meter which measure leaf area rapidly, accurately and non-destructively is introduced in 
[11]. The microprocessor of leaf area meter is ARM9 microprocessor S3C2440. S3C2440 is the hardware core, which has external expanded mass memory, CMOS camera, LCD touch screen and other modules. Embedded operating system Windows CE is selected as operating system. Application is developed with EVC and digital image processing method. Leaf area and perimeter are determined with this leaf area meter. The results show that the leaf area meter has better features of high precision, high accuracy, low cost, easy operation and good portability.

Vision is the most versatile and efficient sensory system. So, it is not surprising that images contribute an important role in human perception. This is analogous to machine vision such as shape recognition application which is an important field nowadays. [12] describes implementation of image processing on embedded platform and an embedded application, a robot capable of tracking an object in 3-dimensional environment. It is a real time operating system (RTOS) based embedded system which will run the Digital Image Processing Algorithms to extract the information from the images. The camera connected on USB bus is used to capture images on the ARM9 core running RTOS. Depending upon the information extracted, the locomotion is carried out. The camera is a simple CMOS USB-camera module which has a resolution about $0.3 \mathrm{MP}$. Video Linux API's provided by kernel are used to capture the image, and then it is decoded, and the required object location is detected using image processing algorithms. The actuations are made so as to track the object. The embedded Linux kernel provides support for multitasking and ensures that the task is performed within the real time constraints. The OS makes system flexible for changes such as interfacing new devices, handling the file system and memory management for storage of data.

\section{III.CONCLUSION}

So in this paper a portable leaf area meter is implemented with a Beagle Bone Black as it is low cost, low power, high speed and portable. This leaf area meter implemented could be used for both laboratory as well as industrial purpose. This method of measuring the leaf dimensions will be very convenient, accurate, damage free, etc. Other than these advantages using this method we could measure the growth rate of the same leaf in a plant since it is a nondestructive method.

\section{REFERENCES}

[1] Dilşad Engin and Mustafa Engin, “ Design of a Plant Leaf Area Meter Using PV Cell and Embedded Microcontroller" in Advances in Materials Science and Engineering Volume 2013 (2013), Article ID 393045, Measuring Technology and Mechatronics Automation (ICMTMA), 2010 International Confe

[2] Chaohui Lu, Hui Ren; Yibin Zhang; Yinhua Shen, "Leaf Area Measurement Based on Image Processing" in Measuring Technology and Mechatronics Automation (ICMTMA), 2010 International Confe.

[3] Application note on line scan imaging basics, Dalsa technology with vision

[4] Bei Optical Encoder design and operation.
[5] Position Fraba, Fraba Inc, 116 Nassau Drive, Lawrenceville, NJ 08648, www.posital.com.

[6] S.Y.Zheng, L.Gui, X N Wang and D Ma, "Real Time Photogrammetry System based on Embedded Aechitecture", International Archives of Photogrammetry, Remote Sensing and Spatial Information Sciences, Volume XL-5, 2014.

[7] Beagle Bone Black 4D 4.3" LCD CAPE, data sheet, document date 13 December 2013, Revision: 1.8 .

[8] Hamamatsu CMOS linear image sensor S11108, datsheet,

[9] Praveen J, MN Shanmukha Swamy, "Low-Transition Test Pattern Generation For Minimizing Test Power In VLSI Circuits Using BIST Technique", International Journal Of Innovative Research In Electrical, Electronics, Instrumentation And Control Engineering, Vol - 2,pp -2029-2035, 2014.

[10] Texas Instruments, User's Gude, Powering AM335x with the TPS65217x, SLVU5511-October 2011-Revised September 2014.

[11] Beagle Bone Black Reference system manual, REV.

[12] Tian, Youwen; Wang, Xiaojuan ,'Development of hand-held leaf area meter based on s3c2440", 10.1109/icemi.2009.5274498, 2009.

[13] Praveen J, MN Shanmukha Swamy, "Minimizing Test Power In VLSI architecture Using BIST Based Low-Transition Test Pattern Generation Technique", International Journal of Electronics and Communications Engineering and Technology, Vol - 5,pp -44-52, 2014.

[14] Sudhir.D.Zaware, Prajwal G. Awade, Chinmay.A.Joshi, R.V.Tornekar, "Image processing algorithms for robotics on embedded system", Proceedings of 11th IRF International Conference, 15th June-2014, Pune, India, ISBN: 978-93-84209-27-8. 